\title{
Jet Dynamics Associated with Drop Impact on Micropillared Substrate
}

\author{
Brooklyn Asai, Anayet Ullah Siddique (D) and Hua Tan *
}

check for

updates

Citation: Asai, B.; Siddique, A.U.; Tan, H. Jet Dynamics Associated with Drop Impact on Micropillared Substrate. Fluids 2021, 6, 155.

https://doi.org/10.3390/fluids6040155

Academic Editor: Mehrdad Massoudi

Received: 23 March 2021

Accepted: 8 April 2021

Published: 12 April 2021

Publisher's Note: MDPI stays neutral with regard to jurisdictional claims in published maps and institutional affiliations.

Copyright: (c) 2021 by the authors. Licensee MDPI, Basel, Switzerland. This article is an open access article distributed under the terms and conditions of the Creative Commons Attribution (CC BY) license (https:/ / creativecommons.org/licenses/by/ $4.0 /)$.
Mechanical Engineering, School of Engineering and Computer Science, Washington State University-Vancouver, 14204 NE Salmon Creek Ave., Vancouver, WA 98686, USA; brooklyn.asai@wsu.edu (B.A.); a.siddique@wsu.edu (A.U.S.)

* Correspondence: hua.tan@wsu.edu

\begin{abstract}
The jetting phenomenon associated with droplet impact upon a hydrophilic micropillared substrate was analyzed in detail using a high-speed camera. Viscosities of the fluids were varied using differing concentrations of glycerol in deionized water. This paper aims to connect similarities between this form of capillary jetting and another well-known jetting phenomenon from the bubble bursting. Both experience a cavity collapse when opposing fluid fronts collide which causes a singularity at the liquid surface, thus leading to the occurrence of jetting. Following processes used to define scaling laws for bubble bursting, a similar approach was taken to derive scaling laws for the dimensionless jet height, jet radius, base height, and radius of the jet base with respect to dimensionless time for the jetting phenomenon associated with the droplet impact. The development of a top droplet before the breakup of the jet also allows the examination of a scaling law for the necking diameter. We find that with the proper scaling factors, the evolution of the jet profile can collapse into a master profile for different fluids and impact velocities. The time dependence of the necking diameter before the jet breakup follows the power law with an exponent of $\sim 2 / 3$. Contrastingly, for other jet parameters such as the radius and height, the power law relationship with time dependence was not found to have a clear pattern that emerged from these studies.
\end{abstract}

Keywords: microfluidics; droplet impact; micropatterned surface; jet evolution; scaling law

\section{Introduction}

Droplet impact is omnipresent and has been observed in both natural and industrial processes. The collision of the liquid droplet and a surface leads to diverse outcomes, such as spreading, partial rebound, deposition, and jetting, just to name a few. In recent years, people have observed that a rapid, thin jet can emerge following the droplet impact on hydrophobic substrates within a range of impact velocities and fluid viscosities [1-3]. The thin jet then breaks up to emit one or multiple satellite droplets that are much smaller than the initial impacted droplet. More recently, our lab has discovered that jetting can occur on micropatterned hydrophilic substrates as well [4]. We have also found that the jet occurring during the receding phase of the droplet impact is caused by the collapse of an air cavity in the center of the droplet, which is congruous with other published research on hydrophobic surfaces. In fact, such jetting phenomenon caused by the inertial focusing capillary flow has also been observed in many other situations [5], such as droplet impact on a liquid, bubble bursting, and droplet pinch-off. The work by Eggers and Villermaux details these mechanisms associated with jetting for many cases [6]. The dynamics of the jet plays an important role in nature and a few engineering applications including aerosol dispersion at the sea surface, additive manufacturing technologies, and the food industry [7-9]. Therefore, it is of significance to study the jet dynamics associated with droplet impact on a solid surface.

Many works have been published on the study of jetting, dating back as early as 1876 when Worthington first published findings about droplet impact on a horizontal plate [10]. 
Only in the last couple of decades, however, has technology such as high-speed cameras allowed further examination into the minute details associated with the formation of a jet [11]. Jetting on a hydrophobic substrate has been well studied and has been found to occur in a certain range of the Weber number for most cases. Following the impact of the droplet on the hydrophobic surface, the droplet spreads and then recoils due to surface tension forces pulling the lamella of the droplet back towards the center. An air-filled cavity is then formed, the shape and decrease in size of which can be described by the Rayleigh-Plesset equation [12]. The edge of the cylindrical-shaped cavity then continues to close in and the collision of the inertially focused fluid fronts results in the occurrence of the singularity leading to ejection of a violent jet. Bartolo [13] was one of the first researchers to report on these phenomena with findings that impact of the droplet on a superhydrophobic surface was found to cause a jet that possessed an axial velocity up to 40 times greater than the impact velocity of the droplet on the substrate. This jetting and droplet emission initiated from droplet impact on the hydrophobic surface has also been observed on many other surfaces, such as hydrophilic substrates [4], natural surfaces [14], razor blades [15], liquid surfaces [16], and oblique hydrophobic substrates [17], and, more recently, discovered to occur on fine mesh [18].

It is well known that the bursting of a gaseous bubble at the liquid surface also produces a jet. As the bubble rises to the surface of a fluid, the previously closed cavity opens at the surface, thus propagating a capillary wave. This capillary wave then oscillates downwards, causing inertial capillary focusing located at the bottom of the bubble cavity, resulting in an eruption of a jet followed by emission of one or multiple satellite droplets due to the breakup of the jet. Ghabache et al. [11], ref. [19] reported on the self-similarities that lead to collapse of the air cavity, thus inducing jetting, and how these properties affect the size of the top droplet. Gañán-Calvo [20] examined the conversion of energy during the jet formation in the bubble bursting and proposed the scaling laws for the axial velocity of the fluid immediately after jet formation and the droplet size of the top satellite droplet from the jet breakup. Brasz et al. [21] and Lai et al. [22] presented an in-depth analysis of the jet evolution following bubble bursting, deriving multiple scaling laws that collapse the jet profile over time governed by the Laplace number. These published results, amongst many other papers, detail the multiple facets of jet dynamics for bubble bursting and droplet impact on many substrates as we understand it today.

In this study, we carried out experiments using millimeter-sized droplets of different fluids hitting a micro-structured hydrophilic surface with different impact velocities. We aim to connect similarities between jetting from the droplet impact and the jetting induced by the bubble bursting. A high-speed camera was used to record the evolution of the jet including initial emergence, rapid growth, and final breakup. An in-house developed MATLAB tool was utilized to process the captured images for automatic measurement extraction of the jet profile, jet speed, and size and speed of any satellite droplets. Scaling laws for the evolution of the jet profile were found by analyzing our collected data over a range of viscosities and impact velocities. These scaling relations were derived by tracking different jet characteristics over time involving dimensions such as the jet tip, base, and necking of the jet. Jet evolution was also characterized by the energy analysis of the initiation and breakup of the jet following the cavity collapse.

\section{Materials and Methods}

\subsection{Experimental Setup and the Substrate}

To capture the droplet impinging upon the hydrophilic substrate, a staging setup was implemented, as seen in Figure 1a. The videos of droplet impaction were taken using a Phantom Miro M310 high-speed camera placed horizontally in line with the staging setup to view the profile of the droplets. The frame rates used for data analysis were 11,000 and $18,000 \mathrm{fps}$. A LED backlight was used to better view the edges of the droplet profile and, in some cases, was adjusted to better view the air cavity as the droplet lamella receded. The droplets were dispensed using a $5 \mathrm{~mL}$ Luer-Lock plastic syringe connected to a New 
Era Pump Systems NE-1000 pump that was programmed to release a $10 \mu \mathrm{L}$ droplet each time. The initial diameter of the droplet was measured as $D_{i}=2.7 \mathrm{~mm}$. Droplets were dispensed from various heights ranging from 120 to $700 \mathrm{~mm}$, which equated to an impact velocity ranging from $U_{i}=0.49$ to $1.17 \mathrm{~m} / \mathrm{s}$ [4]. All experiments were conducted at standard ambient conditions corresponding to a temperature of $298 \mathrm{~K}$ and pressure of $100 \mathrm{kPa}$.

(a)

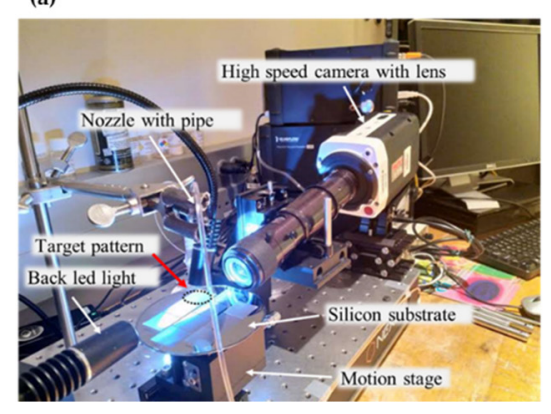

(b)

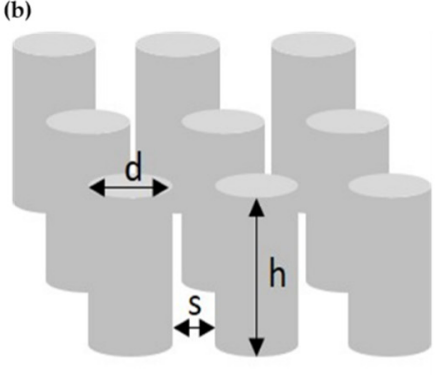

(c)

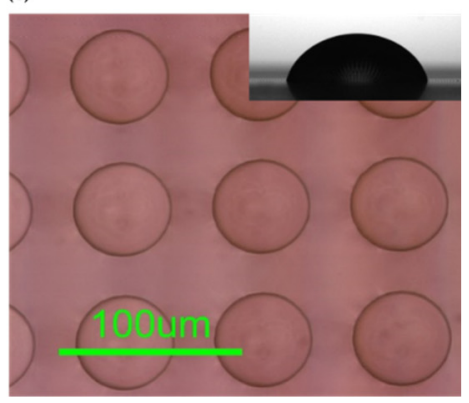

Figure 1. (a) Experimental setup of high-speed camera and staging area, (b) digital rendering of micropillars with diameter, spacing, and height, (c) image of micropillared surface from above taken with a microscope, and inset of side profile of sessile droplet on wafer surface.

The hydrophilic substrate on which the droplets were impacted was a silicon wafer surface. Micropillars on this surface were created using microfabrication processes such as deposition, photolithography, etching, and lift-off. The micropatterned surface had pillars which possessed a spacing $s$ of $21 \mu \mathrm{m}$, diameter $d$ of $52 \mu \mathrm{m}$, and height $h$ of $120 \mu \mathrm{m}$. To include a larger range of fluid viscosity and impact velocity, pillar heights of $100 \mu \mathrm{m}$ were also included in the data analysis. Images of differing perspectives of the silicon wafer surface can be found in Figure 1b,c. Characterization of the silicon surface was completed using a Nikon MM-40 microscope connected to a Nikon DXM 1200 digital camera.

\subsection{Fluid Properties and Dispensation}

Investigating the effects of viscosity on jetting involved the creation of several solutions at different volume percentages of glycerol to water. The volume percent of glycerol ranged from 0 (DI water) to 55\%. In the text and figures, G is used to represent the abbreviated description of the referenced glycerol-water solution. Volume percentages of glycerol were chosen to cover a broad range of viscosities from low cases, such as DI water, to very viscous cases such as the $55 \%$ G solution. Testing the DI water allowed us to be able to compare our results with those found in previous research studies. Mid-range viscosities were taken at random while the high viscosity solution, 55\% G, was found, thus far, to be the threshold beyond which the jetting phenomena of interest did not occur. Table 1 below details the fluid properties recorded for each solution used for droplet impact testing. Included are values for density $(\rho)$, surface tension $(\sigma)$, dynamic viscosity $(\mu)$, static contact angle $(\theta)$, and Ohnesorge number $\left(O h=\mu / \sqrt{\sigma \rho D_{i}}\right)$.

Table 1. Measured fluid properties of glycerol-water solutions used for experimentation.

\begin{tabular}{cccccc}
\hline $\begin{array}{c}\text { \% Glycerol } \\
\text { by Volume }\end{array}$ & $\begin{array}{c}\text { Density } \boldsymbol{\rho} \\
\left(\mathbf{k g} / \mathbf{m}^{\mathbf{3}}\right)\end{array}$ & $\begin{array}{c}\text { Surface } \\
\text { Tension } \boldsymbol{\sigma} \\
(\mathbf{m N} / \mathbf{m})\end{array}$ & $\begin{array}{c}\text { Viscosity } \boldsymbol{\mu} \\
(\mathbf{m P a} \cdot \mathbf{s})\end{array}$ & $\begin{array}{c}\text { Contact } \\
\text { Angle } \boldsymbol{\theta}\left({ }^{\circ}\right)\end{array}$ & Oh \\
\hline 0 (DI water) & 997 & 72 & 0.96 & 51.4 & 0.0023 \\
25 & 1070 & 69.5 & 2.3 & 47.1 & 0.0051 \\
35 & 1100 & 69 & 3.5 & 46.4 & 0.0077 \\
40 & 1113 & 68 & 4.5 & 43.9 & 0.01 \\
50 & 1141 & 67 & 7.7 & 48.5 & 0.017 \\
55 & 1155 & 66 & 10.5 & 49.4 & 0.023 \\
\hline
\end{tabular}


Collection of each droplet video was completed by ensuring the wafer was properly placed in the camera frame on the staging platform, starting the video, and immediately dispensing the droplet from the syringe at the specified height. After the video was captured for a single droplet, the wafer was then cleaned using IPA (isopropanol alcohol), rinsed with DI water, and dried on a hot plate set to the lowest heat setting. This process was then repeated for many different viscous solutions and impact heights.

\section{Results and Discussion}

\subsection{Jetting Phenomena}

Jetting is studied through characterization of its evolution over time. Once a droplet impacts the hydrophilic substrate, the droplet spreads. During spreading, the droplet forms a torus shape and then, once reaching a maximum spreading diameter, begins to recoil. The lamella of the droplet then recedes, with the flow focusing inwards radially, until the liquid walls of the cavity collide, thus resulting in the singularity that initiates the vertical jet. The jet then grows in the axial direction, stretching due to the inertial forces pushing against gravity. Due to surface tension forces fighting this axial growth, a blob forms at the top of the jet and grows in size. At the connection point of this blob to the main jet, the radius of the jet grows thinner, referred to as necking, until eventually the top droplet breaks away. This top droplet, also referred to as a satellite droplet, can grow and break away multiple times in one instance of jetting. An example of this jet evolution can be found in Figure 2. Some common dimensionless numbers used to describe fluid properties and their effects on the presence of a jet are the Weber $\left(W e=\rho U_{i}^{2} D_{i} / \sigma\right)$ and Ohnesorge numbers. $U_{i}$ describes the initial impact velocity. The Ohnesorge number shares a relation with the Weber number by $O h=\sqrt{W e} / R e$, where $R e$ is the Reynolds number $\left(R e=\rho U_{i} D_{i} / \mu\right)$. Both are useful in relating viscous and surface tension forces in the discussion of jet dynamics. Over all experiments, Oh ranged from 0.0023 to 0.023 , We from 9 to 65, and Re from 146 to 3281 for viscosities ranging from 0 to $55 \%$ glycerol by volume.

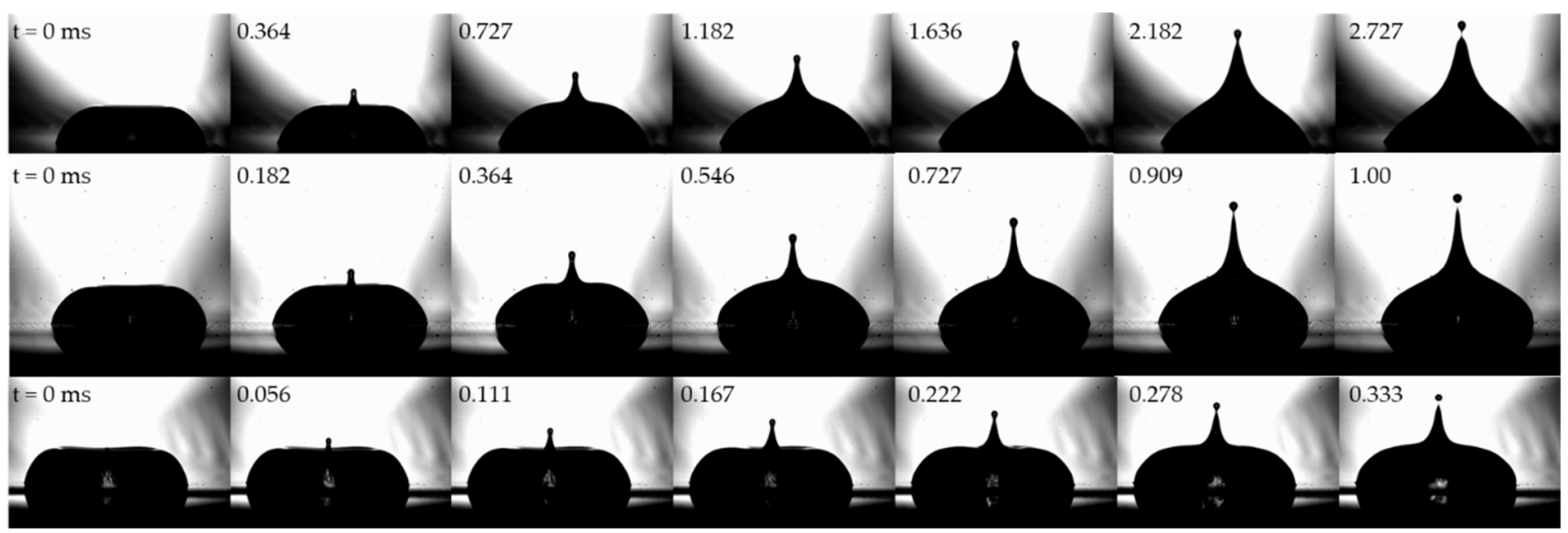

Figure 2. Images showing a typical jet evolution versus time for three different fluids. Top row: $55 \% \mathrm{G}$ solution $(\mathrm{Oh}=0.023$, $\left.U_{i}=0.754 \mathrm{~m} / \mathrm{s}, W e=26.9, R e=223.9\right)$, middle row: $37.5 \% \mathrm{G}$ solution $\left(\mathrm{Oh}=0.0089, U_{i}=0.741 \mathrm{~m} / \mathrm{s}, W e=23.9, R e=553.4\right)$, bottom row: $10 \% \mathrm{G}$ solution $\left(\mathrm{Oh}=0.0029, U_{i}=0.741 \mathrm{~m} / \mathrm{s}, \mathrm{We}=21.4, \operatorname{Re}=1580.3\right)$.

To characterize the jet, a nondimensional process was desired to find the scaling laws for various jet dimensions. The extracted dimensions for each stage of jetting included the radius of the top drop $r_{j}$, maximum jet height $z_{j}$, radius of the jet base $r_{b}$, and height of the jet base from the first frame $z_{b}$ as shown in Figure 3. An extra variable, $n_{r}$, which refers to the necking diameter, was added to further detail the jet breakup. To automate the process of extracting these dimensions from each frame, a MATLAB program was developed. Videos from the droplet experiments were separated frame by frame where each frame represented the profile of the droplet for $1 / 11,000$ th of a second, which is the inverse of the video frame rate 11,000 fps. Importing the frames into the program, the 
images were then cropped and binarized, and the boundaries of the bodies in the image were traced. These data were then refined to eliminate noise inside the droplet and any extraneous bodies found by the program in the individual images. Then, the number of data points extracted was reduced to clean up the profile border so the dimensions could be isolated using further program processing.

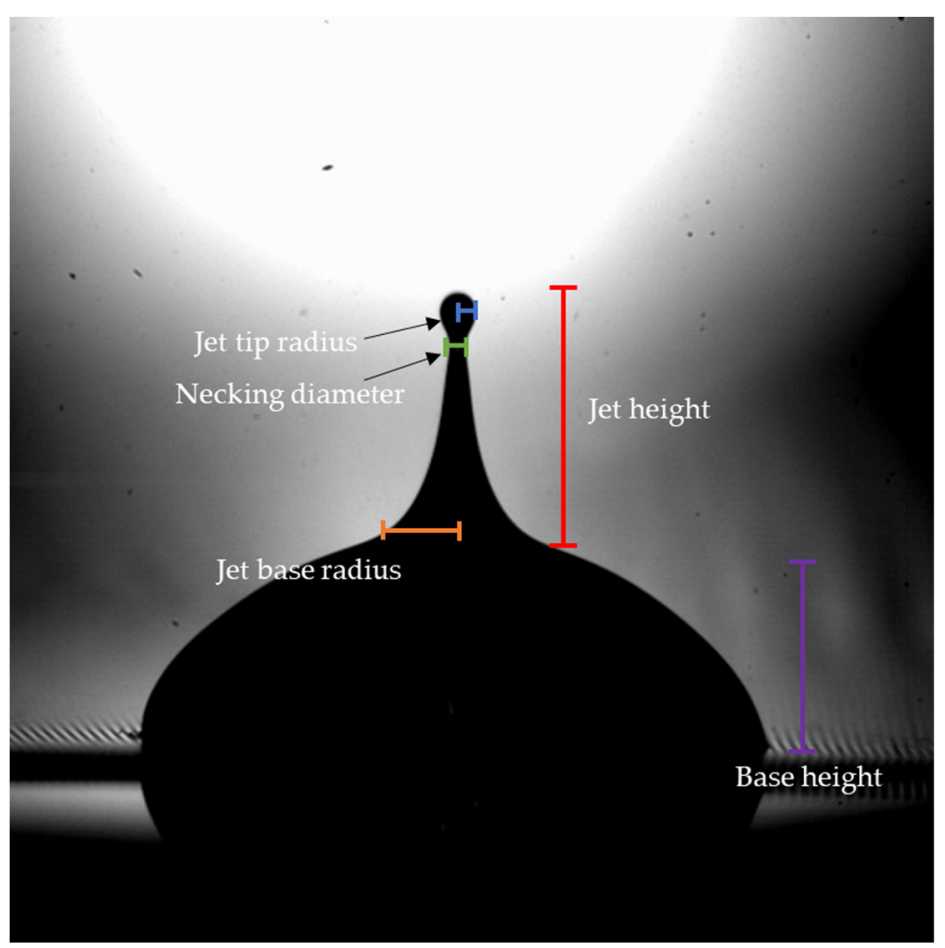

Figure 3. Image of jet dimensions that were extracted with labels.

To find the scaling factor for each jet dimension, nondimensionalized lengths and time were used to derive a power law, and therefore scaling factor. Jet dimensions were normalized by the capillary length, treated as the characteristic length in this system $\left(l_{c a p}=\sqrt{\sigma / \rho g}\right)$. The time for all jet scaling was taken as $\left(t-t_{c}\right) / \tau$, where $t_{c}$ represents the time at cavity collapse before jetting occurs and $\tau$ is the capillary time constant $\sqrt{\rho R_{i}^{3} / \sigma}$. The exception for this was analysis of the necking diameter, where the nondimensionalized time was taken as $\left(t_{b}-t\right) / \tau$, where $t_{b}$ was the time at breakup when the top droplet separates from the jet. After plotting these normalized dimensions extracted from the jet profiles, a power curve fit of the data revealed the scaling factor (SF) for each measurement. From the works of Bartolo and others [13,21,22], we find that the development of selfsimilarities among profiles following jet evolution has a time dependence of $\sim 2 / 3$, giving us a general scaling factor to base our results upon. If the scaling factor of $2 / 3$ holds for all jet dimensions upon the micropatterned hydrophilic substrate, this implies the jet dynamics hold many similarities to jetting phenomena observed on many other substrates such as bubble bursting near a free surface and superhydrophobic surfaces.

\subsection{Trends in Jet Evolution}

In numerical simulations of a bubble bursting jet, researchers have shown that the jet profile exhibits excellent self-similarity and collapses into one to two master curves after rescaling the dimensions of the jet with the characteristic length [21,22]. In order to see if such self-similarity exists in our case, we rescale the jet dimensions with the self-similar length scale $(\sigma / \rho)^{1 / 3}\left|t-t_{c}\right|^{S F}$, where SF is the scaling factor. SF can be found using the method mentioned previously, i.e., the curve fitting of data of the dimensionless jet dimensions (e.g., $\left.r_{j} / l_{\text {cap }}, z_{j} / l_{\text {cap }}, r_{b} / l_{\text {cap }}\right)$ versus dimensionless time $\left(t-t_{c}\right) / \tau$ [21]. Figure 4 shows 
an example of the typical plots of dimensionless jet dimensions versus dimensionless time for a droplet of $40 \% \mathrm{G}$ by volume with an impact velocity of $0.7004 \mathrm{~m} / \mathrm{s}(\mathrm{We}=21.7$, $R e=467.7)$. The scaling factor is extracted using the power law fit for each jet dimension. The jet height grows much faster than the radius of the jet tip, whereas the jet base radius stays nearly constant. It is worth noting that the scaling factor for each jet dimension deviates from $2 / 3$ which is found in a bubble bursting jet [21], among other substrates. Figure $5 \mathrm{a}, \mathrm{b}$ plot the time evolution of the original jet profile and rescaled jet profile, respectively. It can be seen from Figure $5 b$ that the evolution of the jet profile does collapse into a narrow range. Therefore, the jet shape in our study also exhibits self-similarity during the growth, which means that the jet dynamics is governed by capillary-inertial self-similar dynamics.

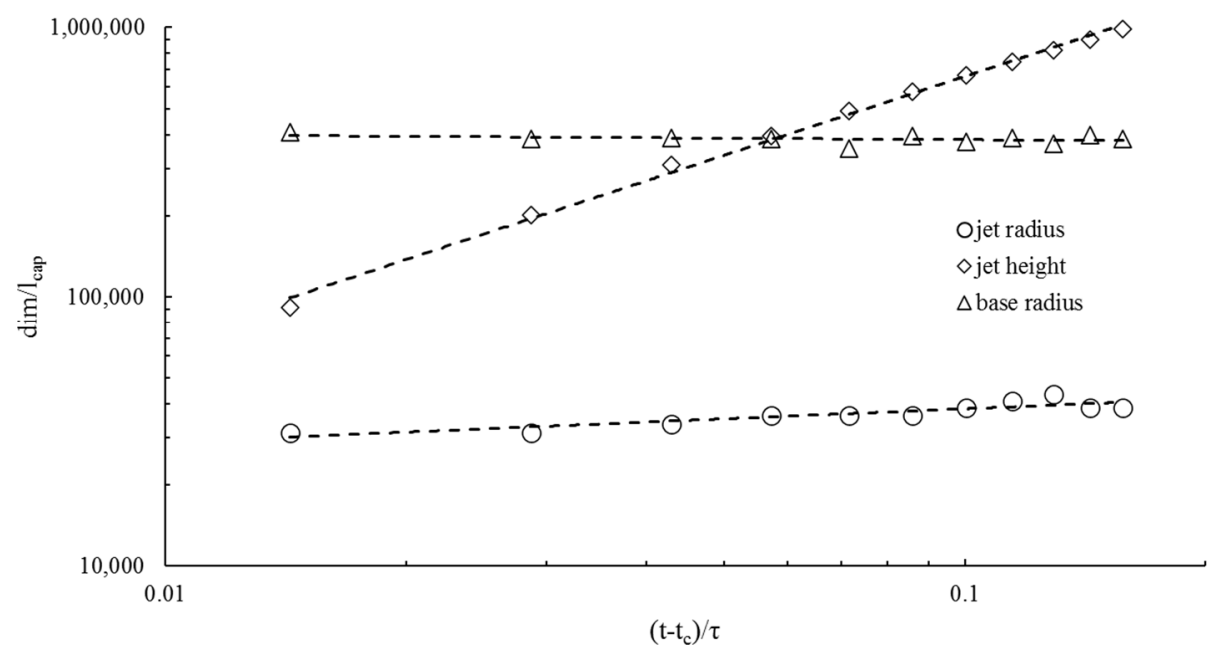

Figure 4. Log-log plot of dimensionless jet measurements versus dimensionless time of droplet of $40 \%$ G solution $\left(U_{i}=0.7004 \mathrm{~m} / \mathrm{s}, W e=21.7, R e=467.7\right)$ for jet tip radius (with power law fit $r_{j} / l_{\text {cap }} \sim(t$ $\left.\left.-t_{c}\right) / \tau\right)^{0.12}$ ), jet height (with power law fit $z_{j} / l_{\text {cap }} \sim\left(\left(t-t_{c}\right) / \tau\right)^{0.97}$ ), and base radius (with power law fit $\left.r_{b} / l_{\text {cap }} \sim\left(\left(t-t_{c}\right) / \tau\right)^{0.02}\right)$.

To study how the viscosity affected the growth of the jet after impact, this process was then repeated for many droplet impact heights and various solutions to create a database of scaling factors for each extracted jet dimension. Table 2 relates the average scaling factor for each dimension over a range of velocities for each studied solution viscosity. As displayed in Table 2, no clear pattern was found in our experimental data compared with the jet evolution from the bubble bursting. Overall trends in the scaling factor exhibited low values for the jet tip radius which had an SF between 0.1 and 0.25 . Jet height grew much more quickly with an average scaling factor of 0.7-1.0. The radius of the jet base was not found to change much with respect to time, and an average scaling factor of 0.01-0.04 was found in this case. We also discovered that the jet dimension scaling, along with the occurrence of a jet after droplet impact, was much more consistent among more viscous solutions that were created in our lab, mainly $40 \%$ glycerol by volume or above. The less viscous solutions, such as DI water and $<10 \%$ glycerol, were found to have much less consistent jet formation and also yielded outlier results when determining a scaling factor for the jet dimensions. For example, a scaling factor of $\sim 6.96$ was found for the radius of the jet tip in DI water, far from any other results determined from more viscous fluids.

Discrepancies of the scaling factors between our study of droplet impact and other studies conducted for bubble bursting can be explained by the different situation leading to the singularity following the cavity collapse in the droplet impact and bubble bursting. For the droplet impact, the surface tension forces pull the rim of the spreading torus-shaped droplet back towards the center during the recoiling stage, causing the fluid to experience almost pure radial flow inwards before the occurrence of the singularity. Contrarily, 
in bubble bursting, the bubble experiences propagating capillary waves that eventually collide and cause the bubble to burst, therefore initiating the singularity that leads to jetting. Additionally, the shape of the velocity profile of the flow across the jet during its growth is different for the jet following droplet impact and the bubble bursting jet. In the case of bubble bursting, it has been proven that the velocity profile across the jet follows a parabolic shape, as detailed in [23]. Contrastingly, in the case of a Worthington jet, such as the jetting observed in our experiments, the flow was found to be uniform across the jet, as discussed in [24]. Due to the concentration of the velocity in the jet following droplet impact being uniformly upwards, this could explain why the scaling factors for jet height that we found across the differing viscosities exhibited a scaling factor of $\sim 1.0$, much larger than the bubble bursting counterpart of $\sim 2 / 3$. The same reasoning can possibly explain the much smaller SF found for the jet tip radius since the parabolic velocity profile of the bubble bursting is the highest in the center of the jet, thus allowing the growth of the top droplet to be much faster, an SF of $\sim 2 / 3$, in comparison to our findings of $0.1-0.25$.
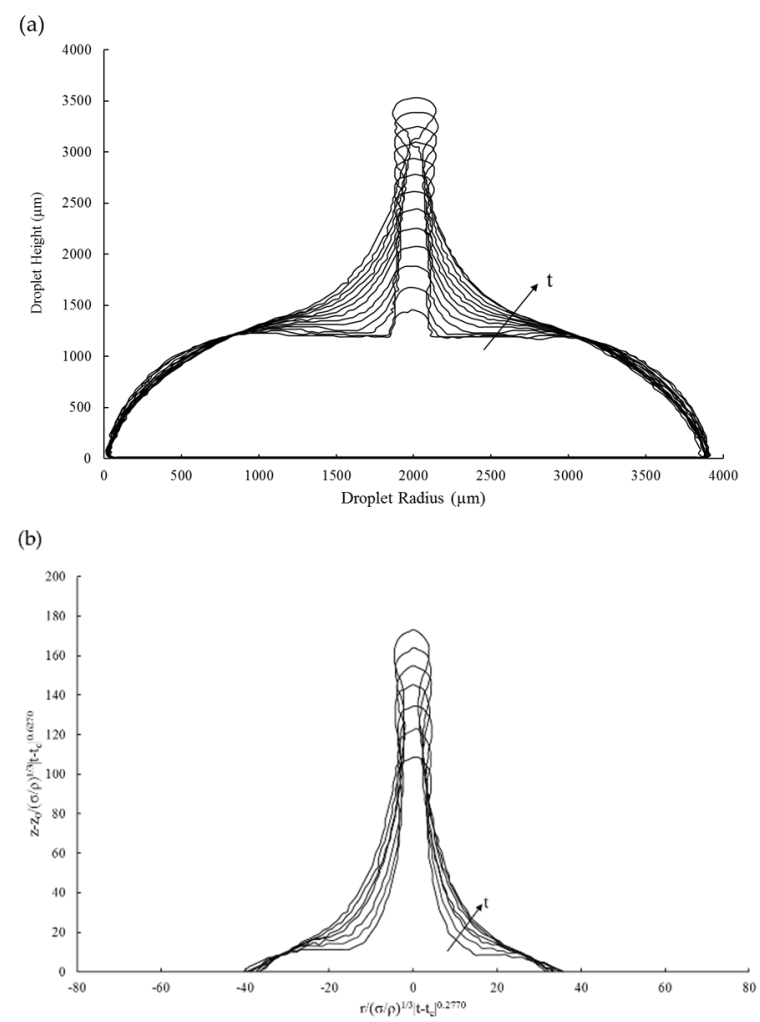

Figure 5. (a) Droplet profiles detailing jet evolution versus time with no scaling applied, (b) same droplet evolution but with scaling applied to show curve collapse. The $35 \% \mathrm{G}$ solution $(\mathrm{Oh}=0.0077$, $\left.U_{i}=0.5943 \mathrm{~m} / \mathrm{s}, W e=15.2, R e=504.3\right)$. Time between curves is $0.091 \mathrm{~ms}$.

Table 2. Average scaling factor of the power law fit for each $\mathrm{G}+\mathrm{W}$ solution extracted by nondimensionalization of droplet profiles for solutions of medium viscosity.

\begin{tabular}{ccccc}
\hline \% Glycerol by Volume & $\boldsymbol{r}_{\boldsymbol{j}}$ & $z_{j}$ & $\boldsymbol{r}_{\boldsymbol{b}}$ & $\boldsymbol{n}_{\boldsymbol{r}}$ \\
\hline DI water & 6.9574 & 0.8423 & 0.5030 & 0.6354 \\
35 & 0.1145 & 1.0061 & 0.0414 & 0.5902 \\
37.5 & 0.2390 & 1.1130 & 0.0200 & 0.7729 \\
40 & 0.1471 & 0.9846 & 0.0190 & 0.6209 \\
50 & 0.2130 & 0.7305 & 0.0520 & 0.8504 \\
55 & 0.1486 & 0.9087 & 0.2266 & 0.6395 \\
\hline
\end{tabular}




\subsection{Breakup of the Jet}

The breakup of the jet in our experiments always takes place at the jet tip, detaching one drop at a time. The breakup occurs as a result of the competition between the capillary retraction of the jet tip, the forming of a blob, and a pressure-driven flow from the cylindrical jet toward the bulbous end [24]. As the jet is stretched inertially, a neck is gradually developed at the connection between the jet and the blob and eventually ruptures, pinching off one or more satellite droplets, as shown in Figure 6. Figure 7 shows the dimensionless necking radius $n_{r} / l_{c a p}$ versus dimensionless time $\left(t_{b}-t\right) / \tau$ leading to the jet breakup following the impact of a $40 \% \mathrm{G}$ solution droplet with an impact velocity of $0.7004 \mathrm{~m} / \mathrm{s}$ (We $=21.7, R e=467.7)$. It is clear that the decrease in the necking radius follows a power law dependence on time with the scaling factor of 0.62 . We apply the same process for all glycerol-water solutions and find the scaling factor in the range of $0.6-0.85$, as shown in Table 2. As the scaling factor is close to $2 / 3$, the jet breakup is controlled by a balance between surface tension and inertial forces [6]. The scaling factors found from our experiments for the necking radius are in general agreement with previously published results from bubble bursting and droplet impact on hydrophobic substrates such as the works of $[13,21,25]$.
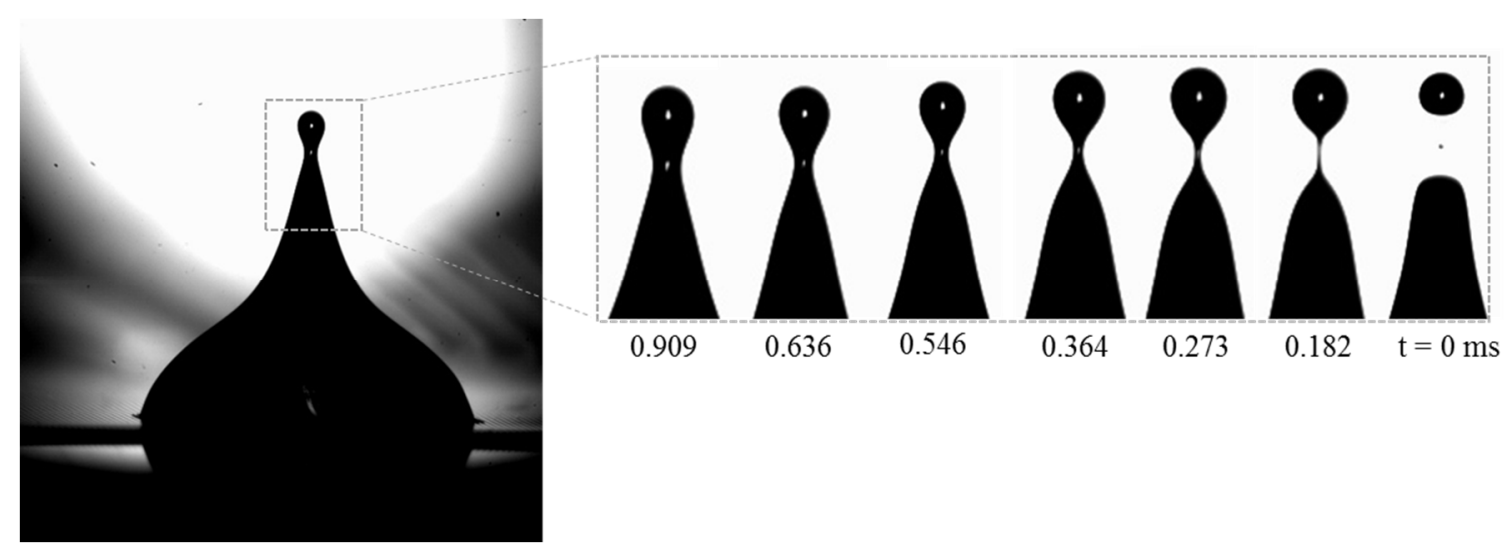

Figure 6. A more detailed image of the necking and jet breakup over time for $50 \% \mathrm{G}$ solution $\left(\mathrm{Oh}=0.017, U_{i}=0.7004 \mathrm{~m} / \mathrm{s}\right.$, $W e=22.6, R e=280.2)$. For scaling of the necking diameter, $t=0$ when the satellite droplet breaks away from the jet tip.

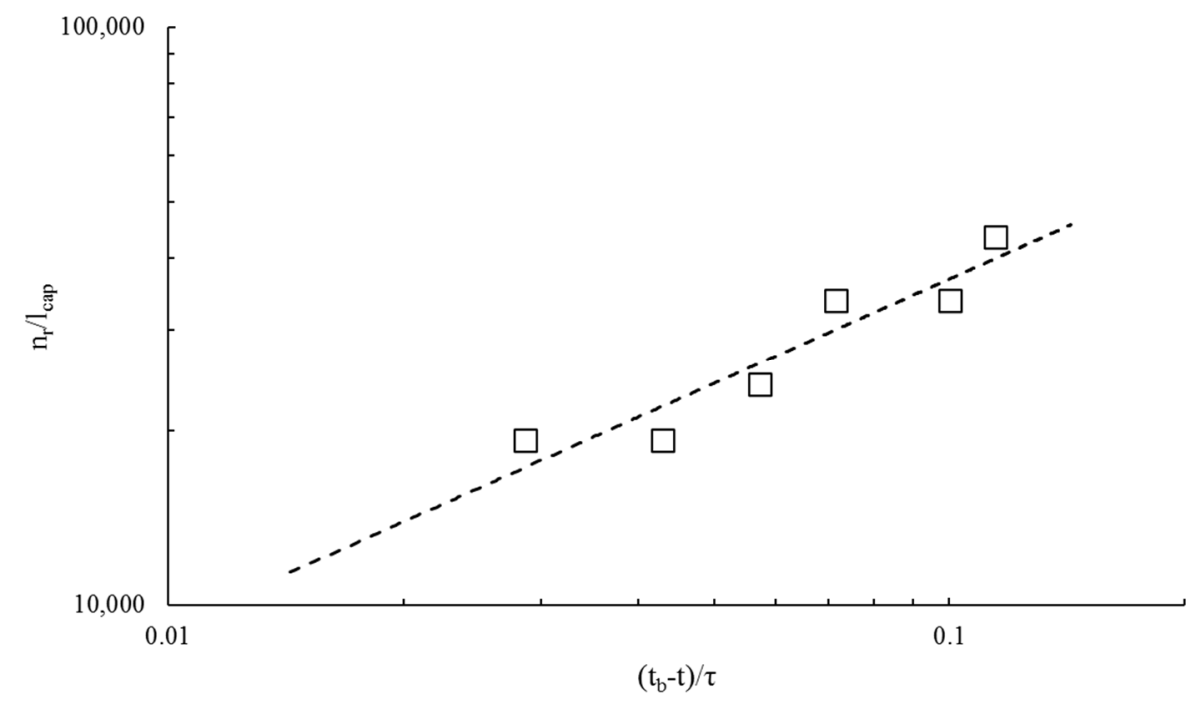

Figure 7. Log- $\log$ plot of dimensionless necking radius $n_{r} / l_{\text {cap }}$ versus dimensionless time $\left(t_{b}-t\right) / \tau$ for $50 \%$ G solution $\left(O h=0.017, U_{i}=0.7004 \mathrm{~m} / \mathrm{s}, W e=22.6, R e=280.2\right)$. The dashed line is the power law fit, $n_{r} / l_{\text {cap }} \sim\left(\left(t_{b}-t\right) / \tau\right)^{0.60}$. 


\subsection{Jet Velocity and Satellite Droplet Size}

When analyzing the jet dynamics associated with the bubble burst, Gañán-Calvo [20] proposed relating the surplus of mechanical energy to the initial jet speed and size of the satellite droplet following jet breakup. In his analysis, the initial jet speed and satellite droplet size were related to a scaling variable $\varphi=\left(O h^{*} / O h-1\right) O h^{-1}$, where $O h^{*}$ is the critical Ohnesorge number beyond which jetting was not found to occur. The $O h^{*}$ previously found in our lab was $\sim 0.028$ [4]. Using the scaling relations proposed by GañánCalvo, we constructed plots to explore any further similarities between the jet initialized by the droplet impact and the bubble bursting jet.

Figure 8 details the comparison of the axial jet velocity immediately after jet initiation with the scaling variable $\varphi$. Here, the initial jet velocity $\mathrm{V}$ is normalized by the viscocapillary velocity $V_{\mu}=\sigma / \mu$. In Figure 9, an energy analysis is shown comparing the ejected top droplet radius, $\mathrm{R}$, and nondimensionalizing it by $l_{\mu}=\mu^{2} / \rho \sigma$. In the paper by Gañán-Calvo [20], the scaling factors for axial velocity and droplet radius were found to be $-3 / 4$ and $5 / 4$, respectively. From our data analysis, the scaling factors for axial velocity and droplet radius were determined to be -0.43 and 1 , respectively. Comparing these two sets of values shows that the scaling of these parameters by energy analysis seems to be relatively similar for both droplet impact and bubble bursting. Again, it was found that as viscosity increased, the similarity and repeatability of the results also increased. An explanation for why these scaling coefficients are much closer than the jet growth scaling could be the time at which the data are extracted, the beginning and end of the jet growth With the velocity analysis, the only velocity extracted is the initial velocity where the jet growth has not experienced much change. Similarly, with the top droplet analysis, the satellite droplet has already broken away from the jet and therefore the radius of this detached droplet has lessened dependency on the jet growth thereafter.

Finally, we related the normalized ejected droplet radius to the normalized initial axial jet velocity, as shown in Figure 10. In [20], these two nondimensionalized lengths were found to have a dependence of $-5 / 3$. From our data, we found a dependence of -1.69 but only for more viscous fluids. For less viscous fluids, classified in this case as solutions containing $<40 \%$ glycerol by volume, the dependency was found to be up to -3.0. For viscous solutions, this seems to be in good agreement with the results from Gañán-Calvo [20].

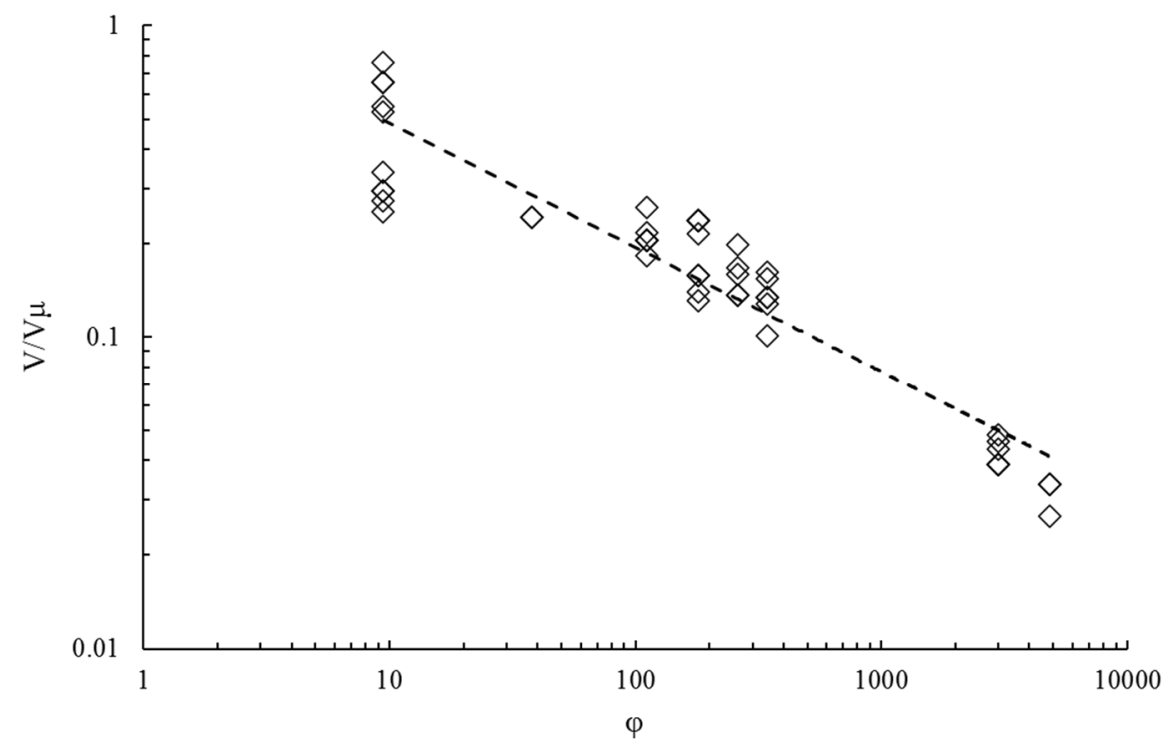

Figure 8. Dimensionless axial jet velocity $V / V_{\mu}$ as a function of $\varphi$ with the power law fit $V / V_{\mu} \sim \varphi^{-0.43}$ for multiple solutions of glycerol volume percentages ranging from 0 to $55 \%$. 


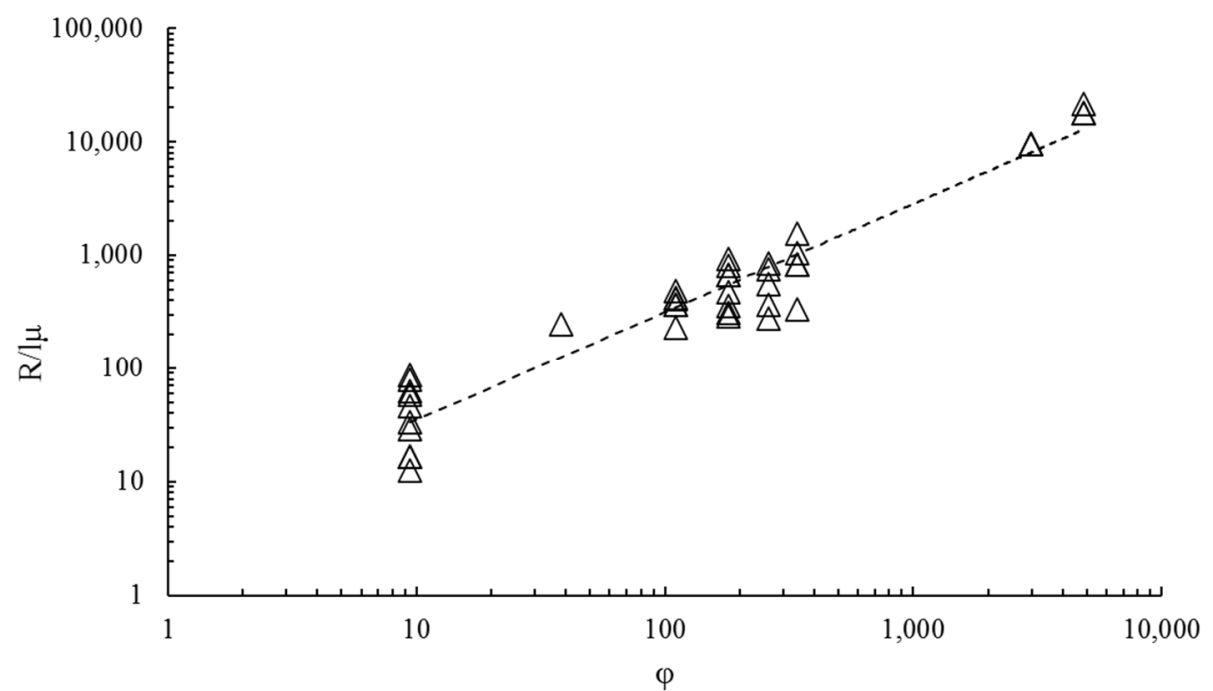

Figure 9. Dimensionless satellite droplet radius $R / l_{\mu}$ as a function of $\varphi$ with the power law fit $R / l_{\mu} \sim \varphi^{1.0}$ for multiple solutions of glycerol volume percentages ranging from 0 to $55 \%$.

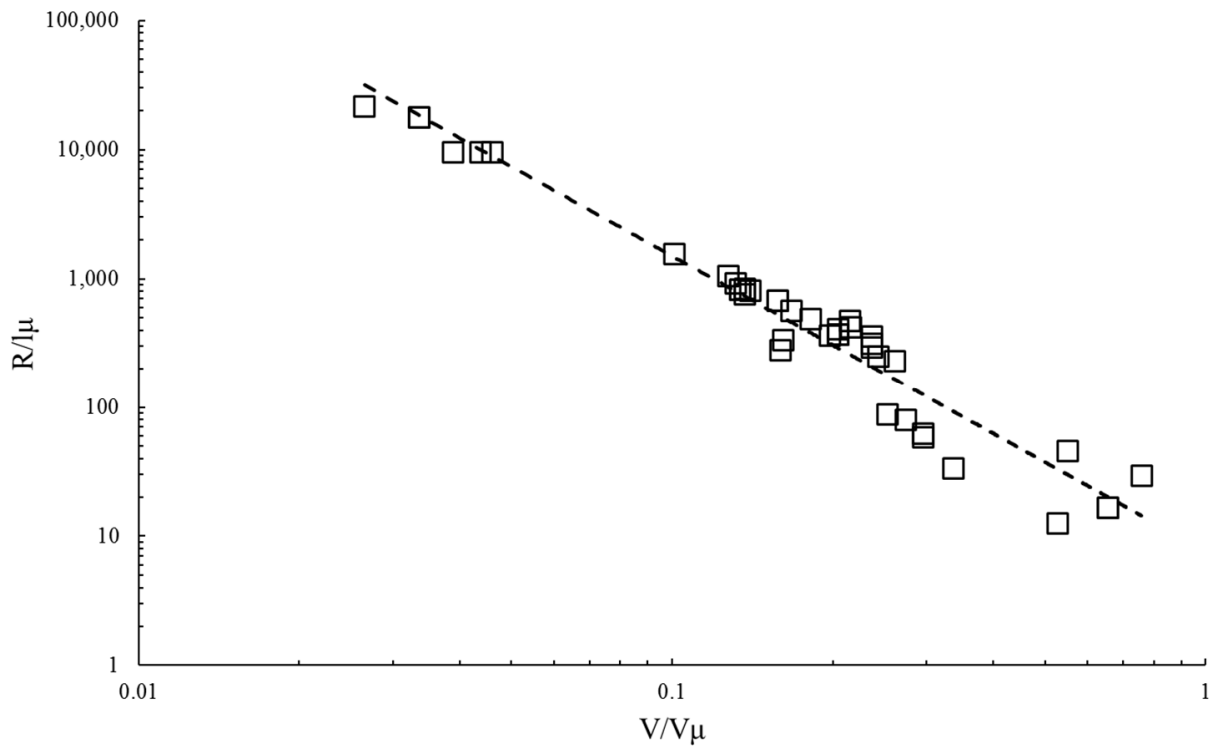

Figure 10. Dimensionless satellite droplet radius $R / l_{\mu}$ as a function of the dimensionless axial jet velocity $V / V_{\mu}$ for more viscous solutions with $>40 \% \mathrm{G}$ with the power law fit $R / l_{\mu} \sim\left(V / V_{\mu}\right)^{-1.69}$.

\section{Conclusions}

In studying the jet dynamics of droplet impact on a hydrophilic substrate, we discovered some key differences and similarities in the jet evolution and breakup in comparison with the jet induced by bubble bursting. Both fluid viscosity and impact velocity were varied in our experiments. The jet height was found to follow a power law for time with a factor from 0.73 to 1.1 , jet tip radius with a scaling factor of $0.11-0.24$, and base radius of the jet with a $0.02-0.5$ scaling factor. These scaling factors are different than the typical scaling factor of $\sim 2 / 3$ obtained from the bubble bursting jet and more hydrophobic substrates. Comparatively, the necking radius of the jet during the breakup process followed a power law dependence on time with a scaling factor ranging from 0.6 to 0.85 , which is close to the expected $2 / 3$ scaling factor found from the jet breakup in bubble bursting. While no clear pattern was found when analyzing the scaling of the other jet dimensions besides the necking radius, we have shown that the jet following droplet impact also exhibits self-similar behavior, as the evolution of the jet profile can collapse into a master profile 
for different fluids and impact velocities using these scaling relations for jet dimensions. The current explanation that we suggest from these data is that for the micro-structured hydrophilic substrate, inertial forces during the collapse of the air cavity in the recoiling phase of the droplet cause a much faster jet growth while slowing the growth of both the jet tip and base radius. Due to the jet velocity profile being nearly uniform following droplet impact and parabolic in nature following bubble bursting, viscous forces have a lessened effect on the axial jet velocity in the case of droplet impact on our substrate. This is a possible explanation for the faster growth of the jet height and slower growth of the bulbous end at the top of the jet. Upon further analysis using an energy balance for jet initialization, we found that the dependences of the dimensionless initial jet velocity and the radius of the first ejected satellite droplet on the nondimensional variable $\varphi=\left(\mathrm{Oh}^{*} / \mathrm{Oh}\right.$ $-1) O h^{-1}$ are $V / V_{\mu} \sim \varphi^{-0.43}$ and $R / l_{\mu} \sim \varphi^{1.0}$, respectively. The scaling factors are in good agreement with the findings from the bubble bursting jet.

Author Contributions: Conceptualization, H.T. and B.A.; methodology, B.A. and H.T.; software, B.A.; validation, B.A., A.U.S. and H.T.; formal analysis, B.A.; investigation, B.A. and A.U.S.; resources, H.T.; data curation, B.A. and H.T.; writing-original draft preparation, B.A.; writing-review and editing, H.T.; visualization, B.A. and A.U.S.; supervision, H.T.; project administration, H.T.; funding acquisition, H.T. All authors have read and agreed to the published version of the manuscript.

Funding: The work is supported, in part, by the National Science Foundation Award CBET\#1701339.

Institutional Review Board Statement: Not applicable.

Informed Consent Statement: Not applicable.

Data Availability Statement: Not applicable.

Acknowledgments: Not applicable.

Conflicts of Interest: The authors declare no conflict of interest.

\section{References}

1. Tsai, P.; Pacheco, S.; Pirat, C.; Lefferts, L.; Lohse, D. Drop Impact upon Micro- and Nanostructured Superhydrophobic Surfaces. Langmuir 2009, 25, 12293-12298. [CrossRef]

2. Chen, L.; Li, L.; Li, Z.; Zhang, K. Submillimeter-Sized Bubble Entrapment and a High-Speed Jet Emission during Droplet Impact on Solid Surfaces. Langmuir 2017, 33, 7225-7230. [CrossRef] [PubMed]

3. Pearson, J.T.; Maynes, D.; Webb, B.W. Droplet impact dynamics for two liquids impinging on anisotropic superhydrophobic surfaces. Exp. Fluids 2012, 53, 603-618. [CrossRef]

4. Siddique, A.U.; Trimble, M.; Zhao, F.; Weislogel, M.M.; Tan, H. Jet ejection following drop impact on micropillared hydrophilic substrates. Phys. Rev. Fluids 2020, 5, 063606. [CrossRef]

5. Zeff, B.W.; Kleber, B.; Fineberg, J.; Lathrop, D.P. Singularity dynamics in curvature collapse and jet eruption on a fluid surface. Nat. Cell Biol. 2000, 403, 401-404. [CrossRef]

6. Eggers, J.; Villermaux, E. Physics of liquid jets. Rep. Prog. Phys. 2008, 71, 036601. [CrossRef]

7. Josserand, C.; Thoroddsen, S. Drop Impact on a Solid Surface. Annu. Rev. Fluid Mech. 2016, 48, 365-391. [CrossRef]

8. Spiel, D.E. More on the births of jet drops from bubbles bursting on seawater surfaces. J. Geophys. Res. Space Phys. 1997, 102, 5815-5821. [CrossRef]

9. Huang, Y.; Jiang, L.; Li, B.; Premaratne, P.; Jiang, S.; Qin, H. Study effects of particle size in metal nanoink for electrohydrodynamic inkjet printing through analysis of droplet impact behaviors. J. Manuf. Process. 2020, 56, 1270-1276. [CrossRef]

10. Worthington, A.M., III. A second paper on the forms assumed by drops of liquids falling vertically on a horizontal plate. Proc. R. Soc. Lond. 1877, 25, 498-503. [CrossRef]

11. Ghabache, É.; Antkowiak, A.; Josserand, C.; Séon, T. On the physics of fizziness: How bubble bursting controls droplets ejection. Phys. Fluids 2014, 26, 121701. [CrossRef]

12. Plesset, M.S.; Prosperetti, A. Bubble Dynamics and Cavitation. Annu. Rev. Fluid Mech. 1977, 9, 145-185. [CrossRef]

13. Bartolo, D.; Josserand, C.; Bonn, D. Singular Jets and Bubbles in Drop Impact. Phys. Rev. Lett. 2006, 96, 124501. [CrossRef]

14. Roy, D.; Pandey, K.; Banik, M.; Mukherjee, R.; Basu, S. Dynamics of droplet impingement on bioinspired surface: Insights into spreading, anomalous stickiness and break-up. Proc. R. Soc. A: Math. Phys. Eng. Sci. 2019, 475, 20190260. [CrossRef] [PubMed]

15. Yamamoto, K.; Takezawa, H.; Ogata, S. Droplet impact on textured surfaces composed of commercial stainless razor blades. Colloids Surf. A: Physicochem. Eng. Asp. 2016, 506, 363-370. [CrossRef]

16. Yarin, A.L. Drop Impact Dynamics: Splashing, Spreading, Receding, Bouncing. Annu. Rev. Fluid Mech. 2006, 38, 159-192. [CrossRef] 
17. Guo, J.; Zou, S.; Lin, S.; Zhao, B.; Deng, X.; Chen, L. Oblique droplet impact on superhydrophobic surfaces: Jets and bubbles. Phys. Fluids 2020, 32, 122112. [CrossRef]

18. Modak, C.D.; Kumar, A.; Tripathy, A.; Sen, P. Drop impact printing. Nat. Commun. 2020, 11, 1-11. [CrossRef] [PubMed]

19. Ghabache, E.; Séon, T. Size of the top jet drop produced by bubble bursting. Phys. Rev. Fluids 2016, 1, 051901. [CrossRef]

20. Gañán-Calvo, A.M. Revision of Bubble Bursting: Universal Scaling Laws of Top Jet Drop Size and Speed. Phys. Rev. Lett. 2017, 119, 204502. [CrossRef] [PubMed]

21. Brasz, C.F.; Bartlett, C.T.; Walls, P.L.L.; Flynn, E.G.; Yu, Y.E.; Bird, J.C. Minimum size for the top jet drop from a bursting bubble. Phys. Rev. Fluids 2018, 3, 074001. [CrossRef]

22. Lai, C.-Y.; Eggers, J.; Deike, L. Bubble Bursting: Universal Cavity and Jet Profiles. Phys. Rev. Lett. 2018, 121, 144501. [CrossRef]

23. Li, T.; Wang, S.-P.; Li, S.; Liu, W.-T. Bubble interactions and bursting behaviors near a free surface. Phys. Fluids 2019, 31, 042104. [CrossRef]

24. Gordillo, J.M.; Gekle, S. Generation and breakup of Worthington jets after cavity collapse. Part 2. Tip breakup of stretched jets. J. Fluid Mech. 2010, 663, 331-346. [CrossRef]

25. Séon, T.; Liger-Belair, G. Effervescence in champagne and sparkling wines: From bubble bursting to droplet evaporation. Eur. Phys. J. Spec. Top. 2017, 226, 117-156. [CrossRef] 